\title{
Caracterización de los efectos de la isquemia normotérmica en la microcirculación renal
}

\author{
E. Lledó García, I. Berenguer García*, G. Pedemonte, D. Rodríguez Martínez*, D. Subirá Ríos \\ C Hernández Fernández, J.F. del Cañizo López* \\ Servicio de Urología-Unidad de Preservación Renal Experimental \\ * Laboratorio de Circulación Artificial. Departamento de Medicina y Cirugía Experimental. \\ Hospital General Universitario Gregorio Marañón
}

Actas Urol Esp 2005; 29 (8): 739-742

\section{RESUMEN \\ CARACTERIZACIÓN DE LOS EFECTOS DE LA ISQUEMIA NORMOTÉRMICA EN LA MICROCIRCULACIÓN RENAL}

Presentamos los resultados obtenidos en un trabajo experimental en el que evaluamos las consecuencias de la isquemia normotérmica en la perfusión hipotérmica $(\mathrm{PH})$ del riñón aislado. Sometimos a un grupo de riñones a PH tras clampaje del pedículo durante $45 \mathrm{~min}$. A otro grupo equivalente de riñones se les perfundió sin isquemia previa. Añadimos a la solución de preservación una cantidad definida de creatinina y canulamos el uréter para recoger el líquido eliminado durante la perfusión y valorar el posible aclaramiento de creatinina $(\mathrm{ClCr})$. Monitorizamos la hidrodinámica de la $\mathrm{PH}$ en tiempo real mediante un sistema informático. En cuanto a los resultados, la resistencia vascular renal (RVR) media de los riñones isquémicos fue mayor, así como el ClCr. Estos dos hechos permitieron inferir, teniendo en cuenta que las diferencias en el estudio histológico fueron mínimas, que los riñones sometidos a isquemia normotérmica experimentan un incremento del tono de la arteriola eferente durante la PH. La PH resultó ser una técnica eficaz para eliminar las consecuencias histológicas de la isquemia. Parece que los cambios microvasculares y bioquímicos producidos durante la $\mathrm{PH}$ pueden ser de causa esencialmente dinámica.

Palabras clave: Trasplante Renal. Preservación Renal. Isquemia.

\section{ABSTRACT \\ NORMOTHERMIC ISCHEMIA EFFECTS IN RENAL MICROCIRCULATION}

We report the results obtained in a experimental work designed to evaluate the consequences of warm ischemia in hypothermic isolated renal perfusion. We perfused a number of kidneys after a period of $45 \mathrm{~min}$ of vascular occlusion. An alternative group of kidneys were perfused without previous warm ischemia. Ureter was canulated in all the procedures and output collected during the HP. Creatinine was added to the perfusion solution initially in order to determine creatinine clearance. HP hydrodynamics was recorded on real time through a computerised system. According to the results, renal vascular resistance as well as $\mathrm{CrCl}$ were higher in ischemic kidneys. Both facts along with minimal differences in pathologic study suggest an increase in vascular tone of efferent-postglomerular arteriole during HP. HP was an adequate technique to minimize histologic consequences of ischemia. Mycrovascular an biochemical changes produced during HP may be produced, essentially, by dynamic causes.

Keywords: Renal Transplant. Renal Preservation. Ischemia.

Trabajo financiado Proyecto F.I.S. (2003-2006) 03/1130. 
$\mathrm{L}^{\mathrm{a}}$ a isquemia normotérmica (donante a corazón parado) se relaciona con una mayor incidencia de disfunción inicial del injerto. Los valores mayores de resistencia vascular renal objetivados en estos órganos se han relacionado en la literatura con alteraciones histológicas (edema intersticial, microtrombosis). En algunos estudios se ha observado que la administración experimental de prostanoides por vía transarterial puede inducir vasoconstricción en la arteriola aferente e hipoperfusión del injerto ${ }^{1}$. No se conoce con exactitud la influencia de estos cambios en la evolución post-implante del riñón, ni el momento exacto de su aparición o la importancia de la técnica de preservación.

\section{OBJ ETIVO}

Estudiar la influencia de la isquemia normotérmica en las modificaciones microvasculares (hidrodinámicas), funcionales (aclaramiento de creatinina) y anatomopatológicas producidas en el riñón aislado durante un período de perfusión hipotérmica.

\section{MATERIAL Y MÉTODO}

16 animales de laboratorio (mini-pig) fueron sometidos alternativamente a nefrectomía simple derecha tras clampaje de pedículo de 45 min o nefrectomía simple izquierda sin isquemia previa. Tras lavado por gravedad con solución UWViaspan $^{\mathrm{R}}$ los riñones se perfundieron durante 60 min en una bomba de perfusión diseñada en nuestro laboratorio y gobernada por vacío. Antes de iniciar la perfusión se añadió a la solución de perfusión (Belzer gluconato) una concentración suprafisiológica de creatinina, y se canuló el uréter. Tras el inicio de la perfusión procedimos al registro hidrodinámico en tiempo real (flujo vascular renal: FVR, resistencia vascular renal: RVR) mediante un transductor arterial de flujo y procesamiento informático de los datos. Se tomaron muestras del líquido eliminado por uréter a los 0 , 15, 30, 45 y 60 minutos para determinar los valores de aclaramiento de creatinina $(\mathrm{ClCr})$. Posteriormente, realizamos estudio patológico de todos los órganos. (Figs. 1, 2 y 3).

\section{RESULTADOS}

Los registros hidrodinámicos de los riñones perfundidos mostraron unos valores de RVR sig- nificativamente más altos en los órganos sometidos a isquemia normotérmica frente a los no isquémicos (1.25 Vs $0.75 \mathrm{mmHg} / \mathrm{ml} / \mathrm{min}$, $\mathrm{p}<0.05)$. El valor medio de $\mathrm{ClCr}$ de los órganos isquémicos fue incluso mayor que el obtenido en los riñones no isquémicos (43 Vs $32 \mathrm{mg}$ ). El estudio histológico mostró cambios mínimos tras la perfusión al comparar los riñones isquémicos y no isquémicos.

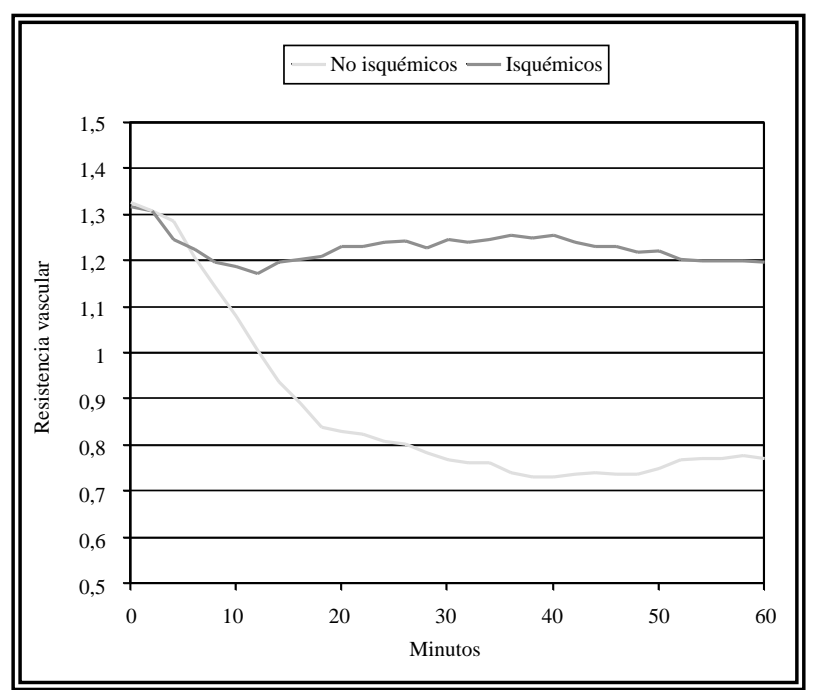

FIGURA 1. Resistencia Vascular Renal Media comparativa entre riñones con/sin isquemia normotérmica previa. Registro en tiempo real durante perfusión hipotérmica del riñón aislado.

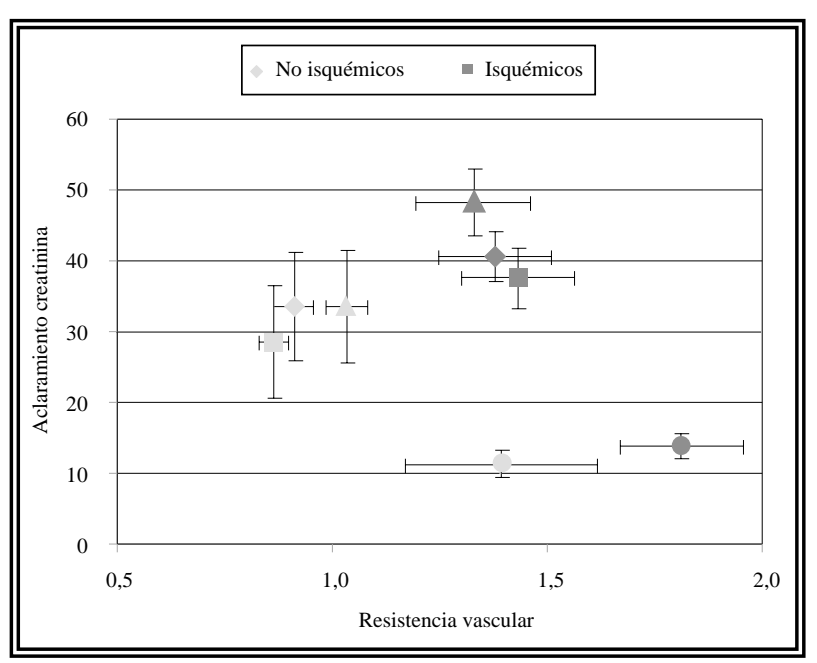

FIGURA 2. Análisis comparativo de aclaramiento medio de creatinina en relación a la resistencia vascular renal efectuado durante la perfusión hipotérmica entre riñones con/sin isquemia normotérmica previa: $\mathrm{ClCr}$ y RVR fueron más altos en los órganos con isquemia. Este hallazgo era compatible con un aumento del tono arteriolar postglomerular. 

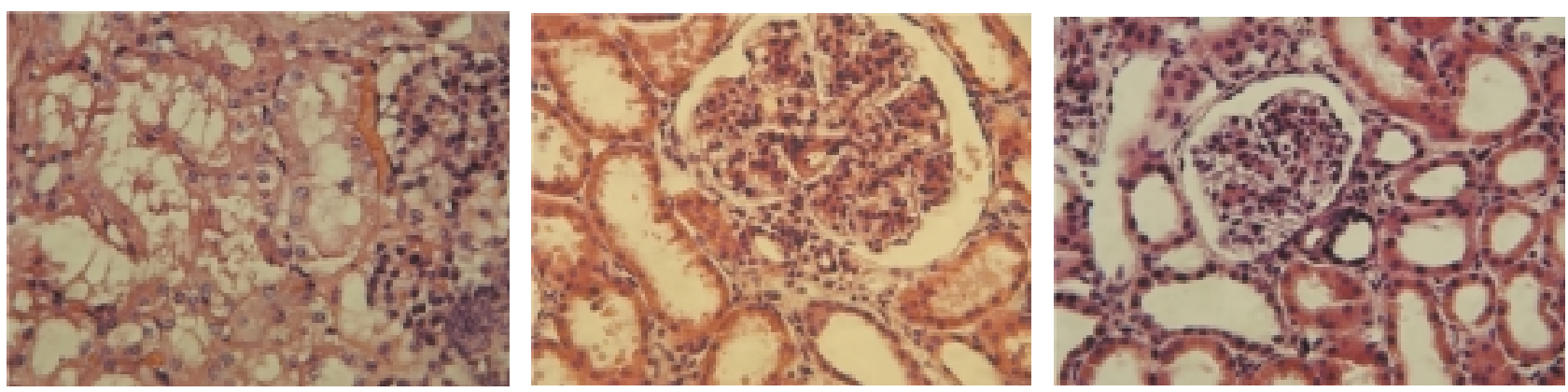

FIGURA 3. Estudio anatomopatológico de los distintos grupos de órganos. A: riñones con isquemia normotérmica previasin perfusión (edema tubulo-intersticial, intensa retracción glomerular, tombosis microvascular); B: riñones con isquemia normotérmica y perfusión (mínima retración glomerular, arquitectura tubular similar a normalidad); C: riñones sin isquemia normotérmica, con perfusión (hallazgos superponibles a grupo B).

\section{DISCUSION}

La composición de las soluciones de preservación/perfusión ha sido en muchas ocasiones, irónicamente, modificada en base a puro empiris$\mathrm{mo}^{2}$. Éste se ha fundamentado en aplicar los principios de la respuesta celular a la isquemia, así como los teóricos menores requerimientos del metabolismo celular durante la hipotermia. El diseño de UW-Viaspan ${ }^{\mathrm{R}}$ produjo un gran salto cualitativo en la preservación mediante hipotermia simple no sólo del riñón sino también de otros órganos abdominales (hígado, páncreas). De la misma forma, la aparición de la solución Belzer-gluconato hizo que la perfusión de riñón aislado mejorara los resultados de esta técnica de preservación, especialmente en el escenario del donante a corazón parado. Sin embargo, en los últimos años se han evidenciado hechos interesantes. Por un lado, que la composición de las soluciones de preservación actualmente existentes no era la óptima, pues sus resultados prácticos en tiempos de isquemia fría prolongados no traducian las expectativas que se habian creado en el terreno experimental ${ }^{3}$. En segundo lugar, que un conocimiento más exacto de los efectos moleculares de la isquemia fría permitiría modificar -al menos experimentalmente- la composición de los medios de preservación, lo que se traduciría en una disminución de la lesión histológica y una mejoría de los resultados funcionales ${ }^{4}$. Parece que publicaciones recientes están renovando el valor que históricamente había tenido la perfusión de riñón aislado en comparación a la preservación por hipotermia simple ${ }^{5}$ ). Por último, es interesante conocer que las observaciones hidrodinámicas durante una perfusión hipotérmica de riñón aislado pueden reflejar no sólo cambios anatómicos secundarios a la isquemia sino un proceso dinámico (¿vascular?) resultante de procesos activos de síntesis de moléculas vasoactivas $^{6-7}$.

Los modelos experimentales permiten inferir procesos moleculares con rangos variables de seguridad. Nosotros administramos a la solución de perfusión una concentración suprafisiológica de creatinina y canulamos el uréter con el objetivo de evaluar, en primer lugar si existía durante la perfusión hipotérmica $\left(4^{\circ} \mathrm{C}\right)$ un proceso de aclaramiento renal similar al desarrollado en la sangre a $37^{\circ} \mathrm{C}$; en segundo lugar, si este extremo se confirmaba, comparar la eficacia de este proceso funcional en presencia/ausencia de una agresión isquémica límite previa a la hipotermia (45 minutos de isquemia normotérmica). A la luz de nuestros resultados podemos inferir la capacidad microvascular glomerular de filtrar la solución de perfusión a $4^{\circ} \mathrm{C}$. Por otro lado, y teniendo en cuenta la mayor RVR media obtenida en los órganos isquémicos, la única vía que permite explicar la obtención de valores de $\mathrm{ClCr}$ iguales/mayores en estos riñones en comparación a los no isquémicos es una mayor contracción de la arteriola eferente, que mantenga/aumente la presión de filtrado glomerular. Si la mayor contracción se produjera en la arteriola aferente -lo que también explicaría el valor más alto de RVR-, la perfusión glomerular y, por tanto, la presión de filtrado glomerular y el $\mathrm{ClCr}$ resultante serían claramente menores. La naturaleza, al menos en gran parte, dinámica-bioquímica del proceso queda corrobo- 
rada en nuestro trabajo por la ausencia de diferencias histológicas significativas entre los riñones perfundidos con/sin isquemia previa. Sin embargo, en un grupo control que evaluamos de riñones con isquemia normotérmica previa y sin perfusión posterior, los cambios patológicos fueron notables.

Nos parecen suficientemente importantes los hallazgos de este experimento ${ }^{8}$, en cuanto que discrepan de los escasos trabajos al respecto. Nos encontramos actualmente depurando los hallazgos bioquímicos, lo que comunicaremos en el futuro.

\section{CONCLUSIONES}

1. Los parámetros hidrodinámicos obtenidos en la perfusión hipotérmica del riñón aislado muestran una mejor situación microvascular en los órganos sin isquemia previa (valores de RVR más bajos). 2. Estas diferencias hidrodinámicas no parecen determinadas por la histología, lo que hace pensar en causas dinámicas. 3 . El valor más alto de la RVR media, junto con la similitud -incluso diferencias- de los valores de $\mathrm{ClCr}$ sugiere que tras la isquemia normotérmica se produce un incremento del tono de la arteriola postglomerular (eferente). 4. La perfusión hipotérmica mejora las características del riñón sometido a isquemia normotérmica minimizando las consecuencias histológicas de la misma.

\section{REFERENCIAS}

1. Takahashi K, Nammour TM, Fukunaga M, Ebert J, Morrow JD, Roberts LJ 2nd, et al.. Glomerular actions of a free radical-generated novel prostaglandin, 8-epi-prostaglandin F2 alpha, in the rat. Evidence for interaction with thromboxane A2 receptors. J Clin Invest 1992;90:136-141.

2. Southard JH. Coffee creamer, the bionic man, and organ preservation. Surgery 2002 Feb;131(2):228-229.

3. Salahudeen AK, Haider N, May W. Cold-ischemia and the reduced long-term survival of cadaveric renal allografts. Kidney Int 2004;65:713-718.

4. Salahudeen AK. Cold ischemic injury of transplanted kidneys: new insights from experimental studies. Am J Physiol Renal Physiol 2004;287:F181-F187.

5. Wight J, Chilcott J, Holmes M, Brewer N. The clinical and cost-effectiveness of pulsatile machine perfusion versus col storage of kidneys for transplantation retrieved from heartbeating and non-heart-beating donors. Health Technology Assessment 2003; Vol 7:No 25.

6. Knes JM, Hansen TN, Gilligan B, Woo $H$, Mangino M, Haworth RA, Southard JH. Loss of endothelium-dependent relaxation in abdominal aorta preserved in a co-storage system. Transpl Int 2004 Nov 17.

7. Lledó-García E, Hernandez-Fernandez C, Díez-Cordero JM, García-Barreno P, del Cañizo-López JF. Hydrodynamic and biochemical effects of isolated hypothermic renal perfusion depending on the pump model and perfusion solution. Transplant Proc 2003 Aug;35(5):1661-1663.

8. E. Lledó, C. Hernández, J.F. del Cañizo. Evaluation of the effects of pulsatile perfusion in kidneys after a period of normothermic ischemia. Abstract 514. American Urological Association Congress. The Journal of Urology 2005;173(4).

Dr. E. Lledó García

Servicio de Urología.

Hospital General Universitario Gregorio Marañón

C/Doctor Esquerdo, 46 - 28007 Madrid

e-mail: ENLLGA@terra.es

(Trabajo recibido el 10 de enero 2005) 